\title{
Precise and predictable mandibuloplasty of asymmetric prominent mandibular angle with aid of 3-dimension printed surgical stent: a case report
}

\author{
Bumjoo Baek, DMD, Jaeman Woo, DMD, Jinyoung Choi, DDS, MD, PhD \\ Department of Oral and Maxillofacial Surgery, School of Dentistry, Seoul National University, Seoul, Rep. of Korea
}

\begin{abstract}
Mandibuloplasty is an increasingly popular procedure among East Asians, because they perceive a wide lower face as a sign of stubbornness and coarseness in personality. Especially for females, such a feature is viewed as masculine and undesirable. Mandibuloplasty is often performed intraorally rather than through an extraoral submandibular approach, to avoid facial nerve injury and visible scar formation. However, the intraoral approach provides limited field of view to a surgeon, thereby increasing the risks of inadequate reduction or asymmetric facial contour. The use of 3-dimension (3D) designed surgical guide serves to overcome the limitations of the intraoral approach and is an invaluable and dependable tool. With the advent of 3D printing technologies, surgical guides are designed preoperatively on computed tomography (CT) scans and they are printed for intraoperative applications, such as asymmetric prominent mandibular angles. The use of CT-designed 3D surgical guides allow for predictable surgery with unequivocally symmetric postoperative skeletal contour. This case report will discuss a successful case of mandibuloplasty utilizing the aforementioned technology.
\end{abstract}

Keywords: angle reduction; asymmetric prominent mandibular angle; computer-aided design; mandibular osteotomy; surgical guide

\section{Introduction}

In the Western population, prominent mandibular angles are viewed as acceptable or even attractive features because Caucasians are mostly dolichocephalic; hence, a wide mandible provides balance to the face, thereby projecting a healthy and attractive image. In contrast, in East Asia, a round, ovoid, or slender facial contour is preferred over a square-shaped face, because the latter is believed to project a stubborn and coarse image. Orientals are mostly mesocephalic or brachycephalic, so the width of a prominent mandible is an imbalance to their relatively short face. East Asian females are increasingly seeking surgery to correct prominent mandibular angles because they believe that such feature projects masculinity rather than femininity.

In angle reduction surgery, surgical planning for the extent and contour of the osteotomy is ambiguous and it is virtually impossible to achieve unequivocally symmetrical shape. Unlike two-jaw surgery, in which the surgical design for repositioning each jaw is determined from model surgery and preoperatively fabricated stents, surgical design for mandibular angle reduction depends on a surgeon's clinical and visual judgement. Facial asymmetry provides an added difficulty in determining the design and precise execution of the osteotomy. With the 
recent development of 3-dimension (3D) printing technology using computed tomography (CT) data, preoperative design of surgical osteotomy in mandibular angle reduction and precise intraoperative replication of the design have become possible. In this case report, we present a case of a 22-year-old female patient who had initially presented with the chief complaints of prominent mandibular angles and facial asymmetry. This patient underwent mandibuloplasty utilizing predesigned 3D printed surgical stents, which facilitated precision and predictability in her surgical outcome.

\section{Case report}

A 22-year-old Korean female patient presented to the Department of Oral and Maxillofacial Surgery, Seoul National University Dental Hospital, with chief complaints of bilateral prominent mandibular angles and a square-shaped face. Upon clinical examination and radiographic analysis, facial asymmetry was diagnosed in addition to prominent mandibular angles (Fig. 1). CT examination revealed more laterally flared left mandibular ramus and angle compared to the right side (Fig. 2). Furthermore, hypertrophic masseter muscles added further width to the lower $1 / 3$ of the patient's face. Precise osteotomy lines for symmetric result was designed using the CT images and surgical guides were preoperatively fabricated with $3 \mathrm{D}$ printing technology to assist in precise replication of the plan. Patient was put under general anesthesia via nasotracheal intubation. Dysport ${ }^{\circledR}$ (Ipsen, Basking Ridge, NJ, USA) injection was done on both masseter muscles (100 unit on each side) (Fig. 3). Intraoral mucosal incision was done on lateral aspects of mandible and a mucoperiosteal flap was elevated to expose the mandibular angle and body of the mandible. The 3D printed angle stents (Fig. 4) were adapted to both sides and osteotomies were executed according to the surgical guides using a piezo-
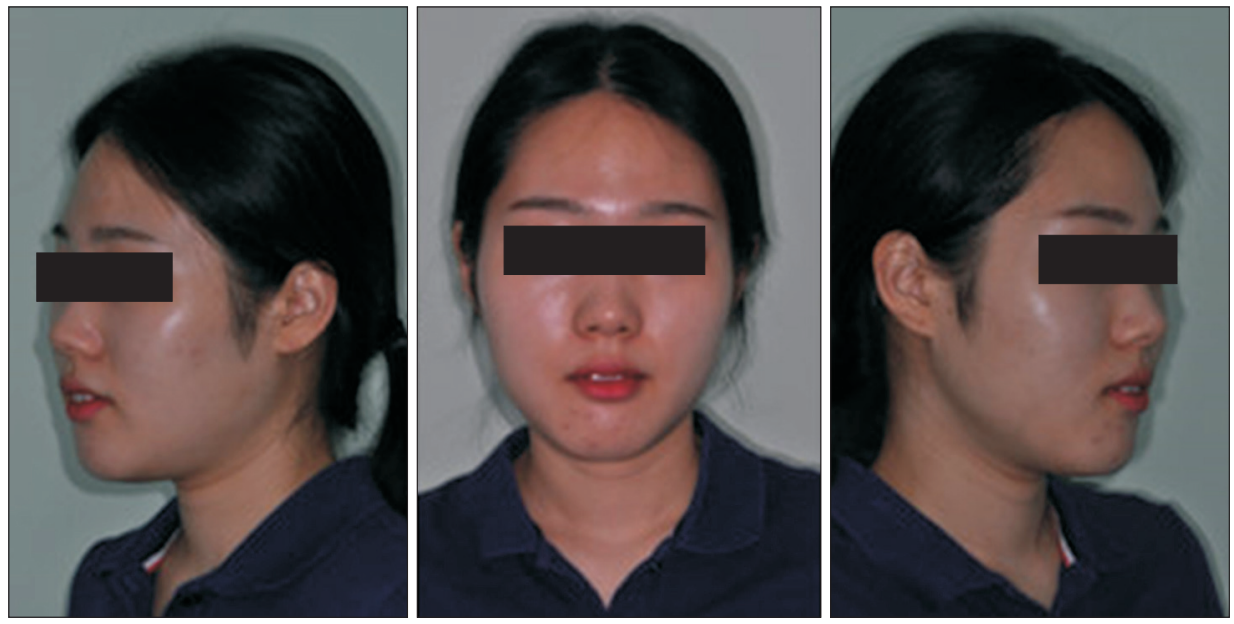

Fig. 1. Preoperative clinical photographs showing facial asymmetry and squareshaped face.
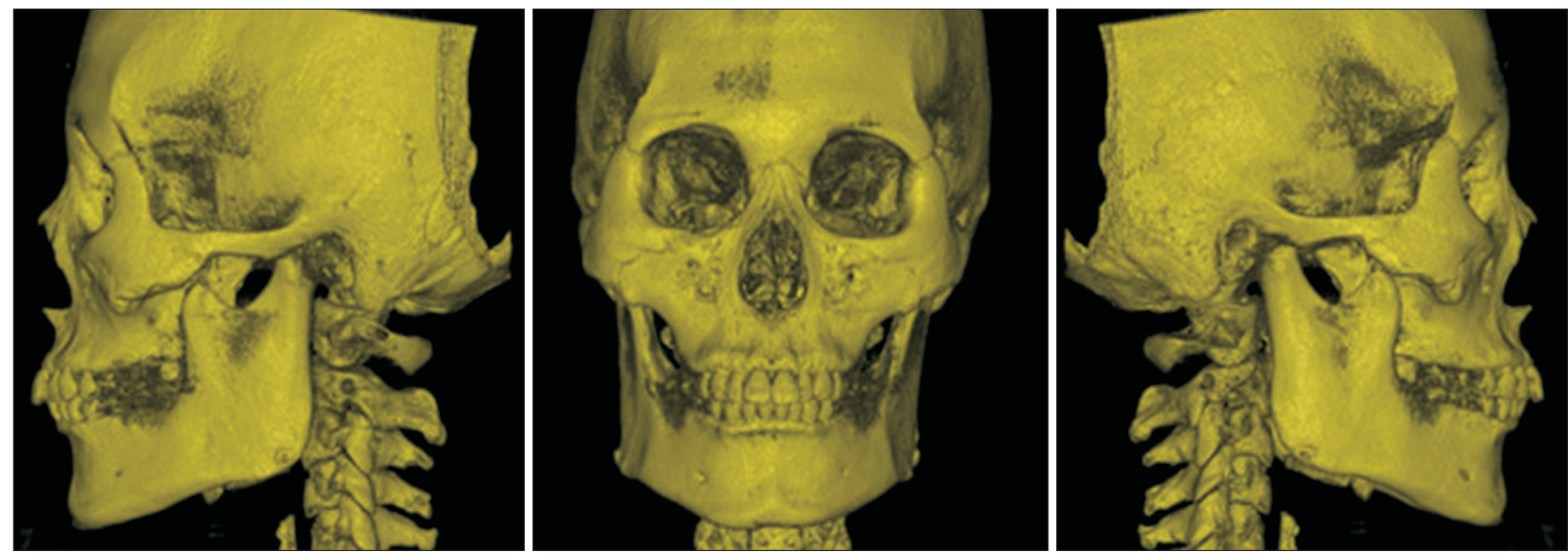

Fig. 2. Preoperative radiographic images. 
electric device, reciprocating saw, and chisel and mallet (Fig. 5). Initial osteotomy incisions were made with the piezoelectric device, which allowed for more control. Then, a reciprocating

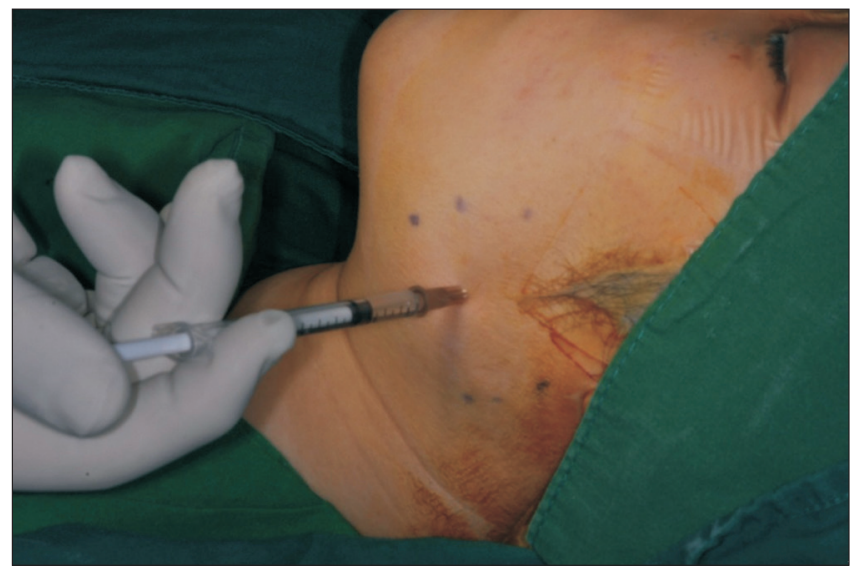

Fig. 3. Dysport ${ }^{\circledR}$ (Ipsen, USA) injection was done on both masseter muscles (100 unit on each side).

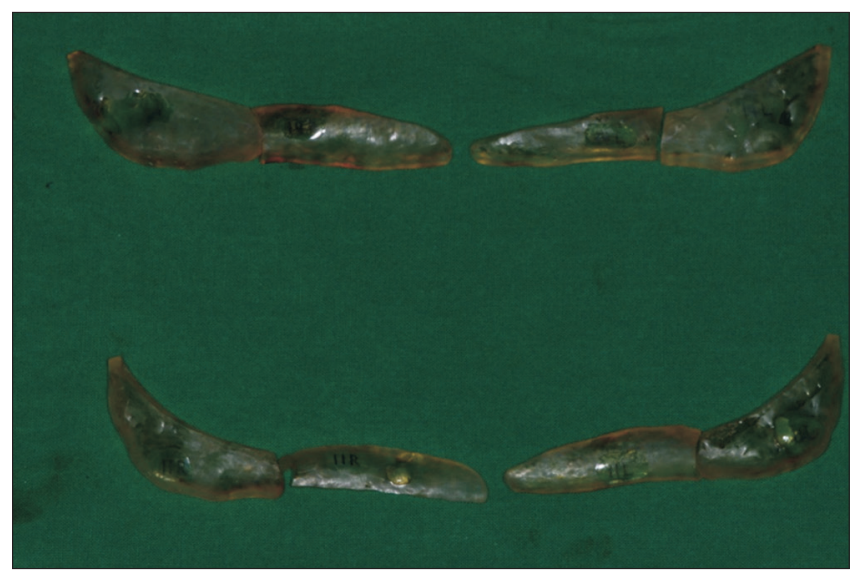

Fig. 4. Mandibuloplasty stents fabricated via 3-dimension printing. saw was used to extend the osteotomy until the lingual cortex was reached and as determined via tactile feel. Once the lingual cortex was reached, osteotomy was completed using the chisel and mallet.

In addition, the left mandibular body corticectomy was done to narrow the lower $1 / 3$ of the face in frontal view. By performing mandibuloplasty and Dysport injection, both skeletal and muscular components of the widened lower $1 / 3$ of the face were resolved in a single operation (Fig. 6). The patient has been followed up in the outpatient clinic for the past twelve months. To date, the patient is satisfied with the postoperative outcome.

\section{Discussion}

Mandibular angle reduction surgeries are mostly done using the intraoral approach because it prevents inadvertent facial nerve injury and visible scar formation. However, the intraoral approach limits visual access of the surgical field, which hinders

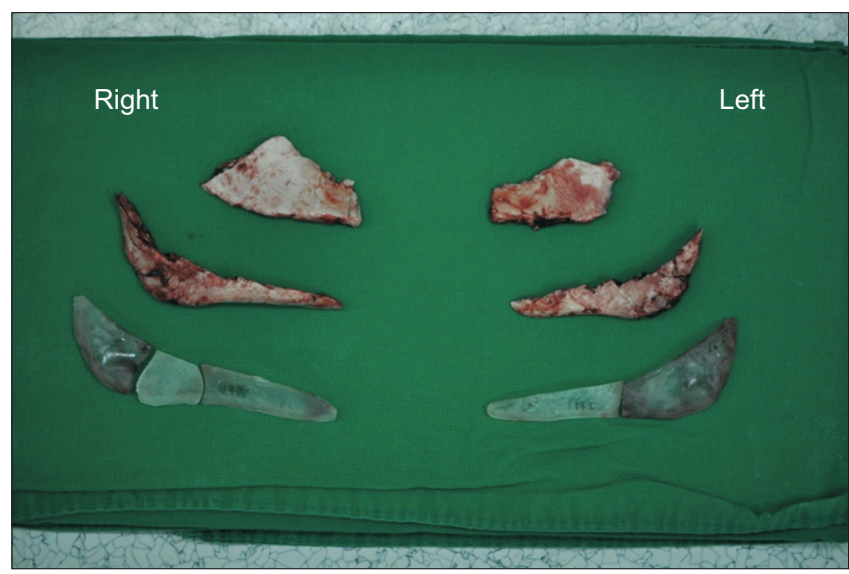

Fig. 5. Resected hard tissues.
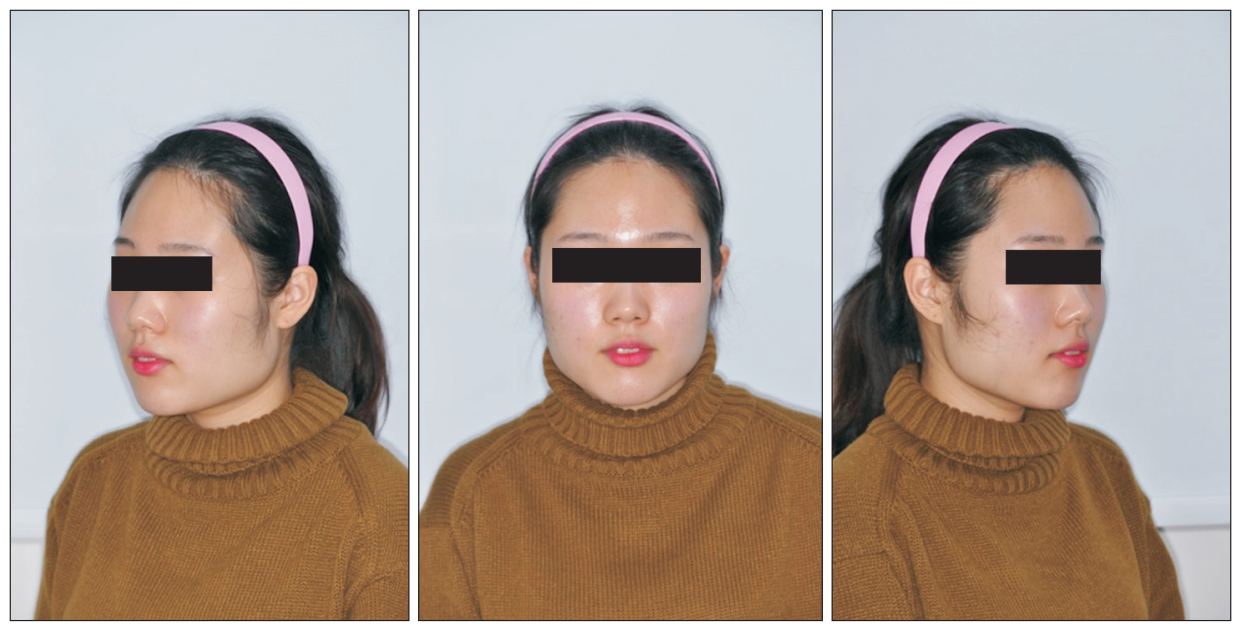

Fig. 6. Postoperative clinical photographs. 
precise determination of appropriate osteotomy lines. Moreover, a surgeon's axis of view is not perpendicular to the mandibular angle and tissue rendition is often limited. Therefore, bleeding from prolonged operation time, unintended subcondylar fracture due to inappropriate osteotomy, postoperative facial asymmetry, inadequate reduction, etc. may ensue, and patient satisfaction may be affected.

To correct prominent mandibular angles, angle reduction surgery has been the treatment of choice. However, the method often resulted in undesirable outcomes such as inadequate symmetry, subcondylar fracture, and unnatural mandibular contour. Most importantly, angle reduction surgery often results in limited esthetic improvement in the frontal view because it does little to reduce the width of lower third of the face. To overcome such a problem, marginal ostectomy of mandibular corpus-angle with corticectomy is indicated [1]. Primary goal of the surgery should focus on the harmonious match of the lower jaw to rest of the face, in both frontal and lateral views. 'Mandibuloplasty' is an inclusive term that encompasses various surgical techniques such as angle reduction surgery, lateral corticectomy. Essentially, mandibuloplasty entails a combination of osteotomy of a mandibular body-angle and corticectomy.

Precision in surgery had depended on skill and experience of a surgeon. Surgical stent could be manually made with the aid of rapid prototype (RP) models. The stent adapted to the angle of mandible serves as a guide for osteotomy. However, manual fabrication of surgical stent is prone to human error and the RP models incur additional cost to the patient.

Precise planning and execution of surgery became possible with the development of computer assisted design and computer assisted manufacture (CAD-CAM) technology and its application in hard tissue esthetic surgery. For example, CAD-CAM osteosynthesis is a new promising method in orthognathic surgery, whereby surgical planning is done through computer software and pre-bent plates serve concurrently as positioning guides for maxilla and rigid fixation, rendering intermediate wafers unnecessary. Time-consuming, error prone preparatory steps such as model surgery may also be bypassed by using this technique.

In this case report, CAD-CAM technology is applied to mandibuloplasty. Preoperative CT scan was assessed and a surgical plan was established virtually. The stent was designed via computer software and printed by the CAD-CAM technique. The 3D printed stent served as an osteotomy guide. This is in contrast to conventional surgery, whereby the result of mandibuloplasty largely depended on the surgeon's clinical and visual judge- ment. This new method, using CAD-CAM technology, is more dependable, predictable, and safe. Conventional intraoral mandibuloplasty was very difficult due to limited operative view and limited space for instrument handling. Lei et al. [2] and Kim and Park [3] reported that they overcame the aforementioned limitations by making a small, inconspicuous incision behind the earlobes and bluntly dissecting through overlying soft tissues to make a tunnel through which a reciprocating saw is handled. In this way, the problems relating to limited space and view were overcome but surgical precision still largely depended on the surgeon. The 3D stents facilitate precision surgery; overcoming limitations such as visual and physical access and alleviating the need for extraoral skin incision.

In the case presented, both muscular and skeletal components of a widened lower $1 / 3$ of the face were resolved in a single operation by combining Dysport injections and mandibuloplasty. Surgical guides with osteotomy lines designed from 3D CT scans improved predictability of symmetric postoperative outcome by allowing for precise intraoperative replication of the plan. Prior to the availability of CAD-CAM technology, osteotomy lines were designed and freehanded by surgeons, resulting in limitations in both precision and predictability. Even with rapid prototype models, surgical guides were designed and fabricated manually. The 3D printed surgical guide serves as an invaluable surgical tool for the precise execution of osteotomy, overcoming limitations such as restricted visual access, and at the same time, enhancing accuracy.

\section{Conclusion}

The 3D printed surgical osteotomy guide is a dependable tool for mandibuloplasty. It assists in precise replication of osteotomy lines designed from preoperative CT data. The technology is especially helpful in cases of prominent angles with concomitant facial asymmetry, wherein the latter poses an additional difficulty in designing appropriate osteotomy lines.

\section{Conflicts of interest}

The authors have nothing to disclose.

\section{References}

1. Hirohi T, Yoshimura K. Lower face reduction with full-thickness marginal ostectomy of mandibular corpus-angle followed by corticectomy. J Plast Reconstr Aesthet Surg 2010;63:1251-9. 
2. Lei R, Wang Y, Xu S, Hong F, Xu M, Wei L, et al. A modified intraoral and extraoral approach osteotomy for the prominent mandibular angle. J Plast Reconstr Aesthet Surg 2017;70:1091100.
3. Kim Y, Park B. Resection of the prominent mandible angle with intraoral and external approach. Aesthetic Plast Surg 2003;27:3842; discussion 43. 